Document downloaded from:

http://hdl.handle.net/10251/64610

This paper must be cited as:

Radicelli García, CD.; Cardona Marcet, N.; Gómez Barquero, D. (2015). A SecondGeneration Digital Terrestrial Television Wireless Return Channel Standard for Providing Internet Connectivity in Rural Areas in Latin America. IEEE Latin America Transactions. 13(9):2837-2844. doi:10.1109/TLA.2015.7350028.

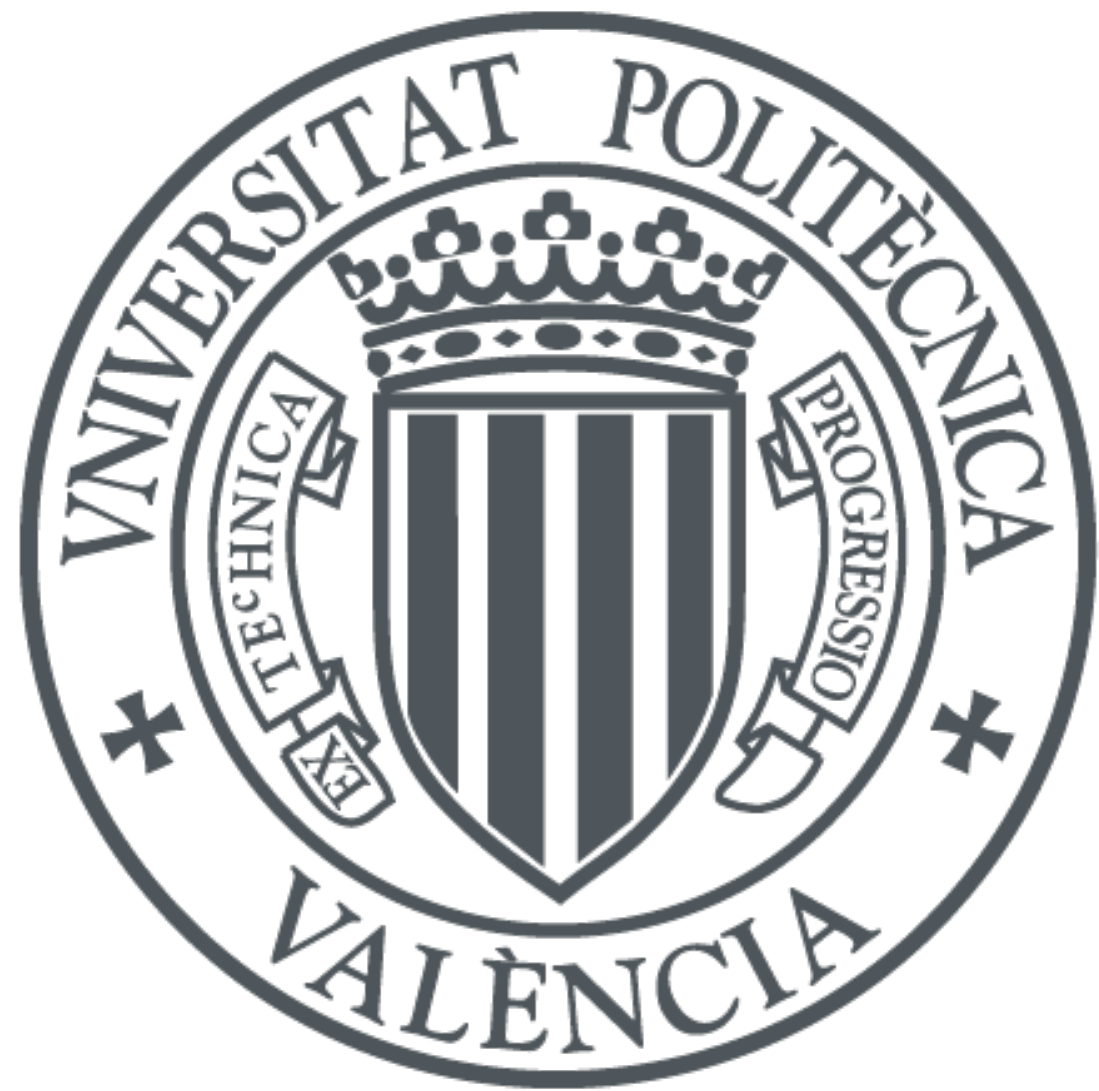

The final publication is available at

http://dx.doi.org/10.1109/TLA.2015.7350028

Copyright Institute of Electrical and Electronics Engineers (IEEE)

Additional Information 


\title{
A Second-Generation Digital Terrestrial Television Wireless Return Channel Standard for Providing Internet Connectivity in Rural Areas in Latin America
}

\author{
C. Radicelli, N. Cardona and D. Gómez
}

\begin{abstract}
This paper proposes the development of a secondgeneration digital terrestrial television wireless return channel for providing universal Internet access in rural areas in Latin America, namely DVB-RCT2. The paper reviews the firstgeneration return channel terrestrial standard DVB-RCT, and discusses which features of the state-of-the-art second-generation digital terrestrial TV standard DVB-T2 should be adopted for DVB-RCT2. Capacity and coverage performance estimations for DVB-RCT2 are provided.
\end{abstract}

Keywords - Digital Terrestrial TV, DVB-RCT, DVB-RCT2, DVB-T2, Internet access, return channel.

\section{INTRODUCCIÓN}

$\mathrm{E}_{\mathrm{d}+\mathrm{sen}}^{1}$ 1 acceso a Internet en zonas rurales de América Latina es deficiente, debido a que la población rural no tiene acceso a esta tecnología o lo hace desde lugares públicos a baja velocidad de conexión. Situación que no sucede con la televisión (TV), que es más popular y accesible que el Internet, la telefonía móvil, e incluso que la radio, teniendo valores de penetración del 90\%, según el último informe de la UIT (Unión Internacional de las Telecomunicaciones) [1].

Latinoamérica está migrando de la TV analógica a la Televisión Digital Terrestre (TDT). Para las emisiones de TDT los países de la región han adoptado en su mayoría el estándar japonés-brasileño ISDB-Tb (Integrated Services Digital Broadcasting - Terrestrial Brasil), a excepción de Panamá y Colombia que utilizan las normas europeas DVB-T (Digital Video Broadcasting - Terrestrial) y DVB-T2 (Terrestrial 2nd Generation) respectivamente, y los países de América Central que usan la norteamericana ATSC (Advanced Television System Committee).

La digitalización de la TV conlleva la liberación de parte del espectro radioeléctrico cuyo uso está previsto para comunicaciones de cuarta generación 4G. Este proceso llamado dividendo digital (DD), corresponde en América (Región 1 de la UIT) a la banda de 700 MHz (canales 52 al 69

C. Radicelli, Universidad Nacional de Chimborazo (UNACH), Riobamba, Ecuador, cradicelli@unach.edu.ec,

N. Cardona, Universitat Politècnica de València (UPV), Valencia, España,ncardona@iteam.upv.es.

D. Gómez, Universitat Politècnica de València (UPV), Valencia, España, dagobar@iteam.upv.es. de TV) [2].Con el despliegue de $4 \mathrm{G}$ en esta banda se espera mejorar el acceso a Internet en zonas rurales, gracias a las velocidades de transmisión que provee y a las mejores condiciones de propagación y penetración que presentan. Sin embargo, es probable que dicho despliegue no sea suficiente para garantizar el acceso en zonas con baja densidad poblacional, además que para acceder a esta tecnología el equipo de usuario tiene un costo elevado. Por lo que se pueden explorar otras tecnologías como WIMAX (Worldwide Interoperability for Microwave Access), la cual presenta características de velocidad y cobertura aceptables de $70 \mathrm{Mbps}$ y hasta $50 \mathrm{Km}$ respectivamente [3], pero con el inconveniente de que los enlaces de microondas se ven afectados por obstáculos como montañas, o factores climatológicos (nieve, lluvia, niebla). Para resolver este problema, se podría utilizar conectividad vía satélite, pero su elevado coste no permitiría universalizar el servicio.

Bajo este contexto, una posible solución para lograr acceso universal a Internet en zonas rurales de Latinoamérica sería la utilización de un canal de TDT con retorno inalámbrico, con lo cual se reutilizaría la infraestructura de TDT para permitir la conexión a Internet disminuyendo los costos de implementación y utilización, puesto que llegaría a todos los hogares que tengan acceso a la TDT. La Tabla I, resume las principales tecnologías que podrían usarse en entornos rurales.

\begin{tabular}{c|c|c|c|c}
\multicolumn{5}{c}{ TABLA I. TECNOLOGÍAS QUE PODRÍAN USARSE EN AMBIENTES RURALES } \\
\hline \hline TECNOLOGÍA & 4G LTE & SATÉLITE & WIMAX & TDT \\
\hline Implantación & Fácil & Fácil & Fácil & Fácil \\
\hline Cobertura & Regional & Continental & Regional & Regional \\
\hline Ancho de banda & Muy alto & Muy alto & Alto & Medio \\
\hline Canal de retorno & Amplio & Amplio & Limitado & Amplio \\
\hline Costos & Alto & Alto & Medio & Bajo \\
\hline
\end{tabular}

Existe una opción en el estándar europeo, DVB-RCT (Return Channel Terrestrial) que proporciona canal de retorno inalámbrico para la tecnología de TDT DVB-T [4]. Sin embargo esta nunca llegó a desplegarse comercialmente debido al deficiente apoyo de la industria, a la oposición de los operadores de telefonía móvil, y a que se necesitan cambios en la regulación del uso del espectro de radiodifusión para su uso.

En este artículo se propone reimpulsar y mejorar el canal de retorno UL (uplink), desarrollando una segunda-generación del estándar DVB-RCT, DVB-RCT2 (Return Channel Terrestrial 2nd Generation), para lo cual se utilizará los 


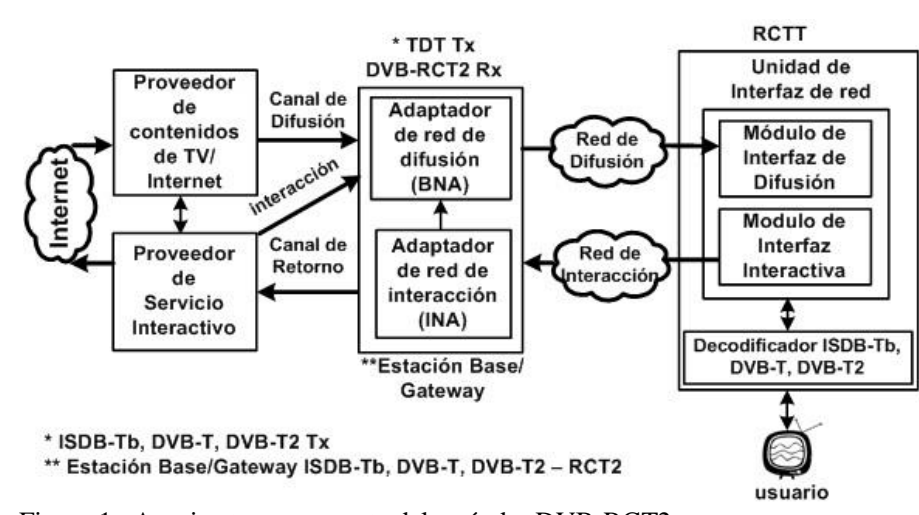

Figura 1. Arquitectura propuesta del estándar DVB-RCT2 para proveer servicios de Internet a zonas rurales.

principios de DVB-RCT y los avances tecnológicos de DVBT2 para aplicarlos en el UL. DVB-RCT2 podrá ser utilizado en DL con cualquier tecnología de TDT (ISDB-Tb, DVB-T o DVB-T2), si se usan el transmisor y decodificador (set-topbox) adecuados, permitiendo además que la difusión de TV en DL y la petición de contenidos de Internet por parte de los usuarios en UL, sean completamente independientes uno del otro, no existiendo cruce de señalización entre dichos medios. A diferencia por ejemplo de otras tecnologías como TDT híbrida que funciona solamente en el estándar europeo DVB, utilizando la tecnología HBB (Hybrid Broadcast Broadband), en donde en el canal de difusión (Broadcast), se emite una señalización específica, la cual permite la directa localización de los contenidos del emisor en el canal de acceso a Internet (Broadband), necesitando de manera forzosa la sincronización de contenidos de ambos canales. Cabe destacar que para el caso de estudio de esta investigación en el Ecuador, se usará ISDB-Tb en el DL. La Fig. 1 ilustra la arquitectura RCT2.

La estructura del artículo es la siguiente. En la Sección 2 se explica la arquitectura, características y prestaciones de la tecnología DVB-RCT. En la Sección 3 se describen las novedades de DVB-T2 con respecto a DVB-T. La Sección 4 se enfoca en el diseño del nuevo estándar, tomando como referencia las nuevas funcionalidades de T2. En la Sección 5 se evalúan las prestaciones que tendría RCT2. La sección 6 se encarga de la estimación de planificación de redes de TDT. En la sección 7 se describe los resultados de dicha planificación. Finalmente en la sección 8 se presentan las conclusiones.

\section{TECNOLOGÍA DVB-RCT}

DVB-RCT fue el primer sistema en el que se propuso OFDMA (Orthogonal Frequency-Division Multiple Access) para el acceso concurrente de múltiples usuarios dentro de un canal de radiofrecuencia (RF) [5]. Utiliza DVB-T para el DL, y la propia tecnología DVB-RCT para el UL. Al estar basado en DVB-T, DVB-RCT reutiliza algunas funcionalidades de aquél, como paquetes MPEG-2 TS (Transport Stream), mecanismo FEC (Forward Error Correction) basado en la concatencación de un código Reed-Solomon (RS) con un código convolucional, y modulaciones QPSK, 16QAM y 64QAM. Pero DVB-RCT introdujo además: (i) turbo-códigos, (ii) segmentación de banda, (iii) modulación DAAM (Dynamically-Assignable Adaptative Modulation), (iv) control de potencia, y (v) entrelazado temporal (Time Interleaving).
Los turbo-códigos reducen la relación portadora a ruido CNR (Carrier-to-Noise Ratio) aproximadamente en $1.5 \mathrm{~dB}$ con respecto a la utilización del código convolucional. La segmentación de banda facilita el acceso al espectro utilizando segmentos de frecuencia libre o cualquier hueco en el espectro desde $1 \mathrm{MHz}$. La modulación adaptativa DAAM permite utilizar una modulación y codificación (MODCOD) específica por usuario, maximizando la velocidad de transmisión según las condiciones de recepción. El control de potencia es similar al de los sistemas celulares, asegurando que los equipos de usuario transmitan con la potencia mínima. El uso de entrelazado temporal brinda mayor robustez frente a la interferencia impulsiva (niveles de interferencia muy altos en muy cortos periodos de tiempo), asegurando de esta manera que el área de cobertura de las señales OFDMA en UL quede balanceada con la cobertura provista por la TDT en DL, con lo que se reduce la necesidad de nuevas instalaciones.

\section{A. Capa Física}

DVB-RCT especifica dos tamaños de FFT (1K y $2 \mathrm{~K}$ ), tres distancias entre portadora CS (Carrier Spacing) CS1, CS2 y $\mathrm{CS} 3$, con 1, 2 y $4 \mathrm{kHz}$ de separación respectivamente, que implican un tamaño de celda máximo distinto y brindan diferente resistencia al efecto Doppler cuando el RCTT está en movimiento. Presenta tres esquemas de modulación QPSK, 16QAM y 64QAM, cuatro intervalos de guarda (GI - Guard Interval) 1/4, 1/8, 1/16,1/32; dos tasas de codificación 1/2 y 3/4.

DVB-RCT permite dos tipos de conformadores de señal para proveer inmunidad contra la interferencia entre portadoras ICI (Inter-Carrier Interference), e interferencia entre símbolos ISI (Inter-Symbol Interference): Nyquist y rectangular. El conformado de Nyquist usa filtrado de Nyquist en tiempo sobre cada portadora, brindando inmunidad contra jammers. Es adecuado para tamaños de celdas grandes en donde los usuarios están alejados unos de otros. Por su parte el conformado rectangular usa una disposición ortogonal de las portadoras y un GI de duración 1/4, 1/8, 1/16, 1/32, y combate los efectos de la propagación multicamino, siendo adecuado para celdas pequeñas donde puede existir solapamiento del espectro. El uso de uno u otro conformador es estrictamente exclusivo, no siendo utilizados al mismo tiempo en UL.

Por su parte, el canal es dividido en una malla de ranuras de tiempo/frecuencia. En cada instante un RCTT puede usar diferentes portadoras OFDM simultáneamente para transmitir, a esto se lo conoce como estructura de ráfaga (BS - Burst Structure) y es la unidad de transmisión básica asignada a los RCTTs. El estándar define tres tipos con un número de portadas que a su vez están estructuradas por subcanales. BS1 con 1 , BS 2 con 4 y BS3 con 29 portadoras, con 1708, 427 y 59 subcanales respectivamente, esto para una FFT 2K. Además el canal presenta una estructura repetitiva provista por dos tipos de tramas de transmisión ( $T F$ - Transmission Frames): TF1 que organiza el canal en el dominio del tiempo para transmitir símbolos nulos, aleatorios y de usuario; y TF2 que lo hace en el dominio de la frecuencia para transmitir símbolos de propósito general. TF1 es usado con BS1 y BS2 utilizando conformado de señal rectangular o de Nyquist, mientras que TF2 se usa con BS2 y BS3 utilizando conformado rectangular. 
La combinación de BS y TF constituyen tres esquemas de acceso al medio (MAS - Medium Access Scheme); MAS1 (TF1-BS1) o acceso de tasa fija, MAS2 (TF1-BS2) o acceso de contención, y MAS3 (TF2-BS3) o acceso de reserva, aunque existe un cuarto esquema que no tiene que ver con esta combinación llamado esquema de sincronización.

\section{B. Capa MAC (Medium Access Control)}

El usuario envía las peticiones a través del Módulo de Interfaz Interactiva hacia el adaptador de red de interacción INA (Interactive Network Adapter) en la estación base, y esta responde a las peticiones desde el BNA (Broadcast Network Adapter), hacia el módulo de Interfaz de Difusión del RCTT.

La capa MAC en el UL proporciona seguridad y compartición del canal entre los RCTTs y la estación base. El INA ejecuta el proceso controlador de la capa MAC para asignar los recursos de subida (ranuras de frecuencia y tiempo) a los RCTTs. Dicho controlador realiza tres procesos fundamentales: sincronización inicial, mantenimiento de la sincronización, y asignación de recursos.

La sincronización inicial para cada RCTT se realiza en tiempo, potencia y frecuencia. Para sincronizarse en tiempo y potencia cada RCTT demodula la señal de difusión DL que provee la estación base obteniendo información sobre la operación de la red, luego de lo cual transmite un código aleatorio de los 32 definidos para tareas de inicialización, mantenimiento o solicitud de ancho de banda en el subcanal de servicio (subcanal de subida predefinido por el controlador MAC mediante el cual entra en la red, y que luego sirve para para mantener la sincronización durante la conexión y para solicitar ancho de banda). Dichos códigos son CDMA (Code Division Multiple Access) por lo que la estación base es capaz de identificar varios códigos recibidos al mismo tiempo. La sincronización en frecuencia se realiza al final y depende de la señal emitida por la estación base. El controlador MAC define cuatro modos de acceso en el UL; (i) Sincronización, para sincronizar inicialmente al RCTT y mantener la sincronización durante toda la conexión; (ii) Tasa fija, para asignar al RCTT una serie de ranuras cuando se establece la conexión; (iii) Contención, para enviar mensajes MAC en las ranuras no reservadas que el INA identifica en el DL; y (iv) Reserva, para conceder a un RCTT ranuras mediante mensajes MAC o por motivos de sincronización.

El controlador MAC continuamente monitorea el UL, asignando el MODCOD que deberán tener los RCTTs por conexión y verificando que estén dentro de los umbrales de sincronización predefinidos; si se detecta algún equipo fuera de estos parámetros, empieza un proceso de recalibración.

Una vez efectuada la sincronización inicial mediante la codificación CDMA, se establece una conexión entre el RCTT y la estación base, la misma que envía en DL un reconocimiento al RCTT para identificarlo. Luego de este proceso se utiliza OFDMA para el acceso de los múltiples RCTTs dentro del canal RF. Para solicitar ancho de banda (ranuras) el RCTT envía un mensaje MAC utilizando los esquemas de acceso al medio. Dichas ranuras le son asignadas en DL mediante los subcanales de datos.

\section{TeCnOlogía DVB-T2}

DVB-T2 presenta numerosos avances tecnológicos frente a DVB-T entre los que destacan [6], (i) mecanismos de corrección de errores FEC: LDPC más BCH, (ii) tuberías de capa física PLP (Physical Layer Pipes), (iii) entrelazado temporal, (iv) constelación 256QAM y constelaciones rotadas, (v) codificación espacial MISO (Multiple Input Single Output) Alamouti, (vi) diferentes patrones de portadoras piloto PPP, (vii) tamaños de FFT de $16 \mathrm{~K}$ y $32 \mathrm{~K}$ con los modos de portadoras extendidos, (viii) mecanismos de reducción de la potencia de pico de la señal PAPR (Peak-to-Average Power Ratio): técnicas de reserva de tonos TR (Tone Reservation) y extensión activa de la constelación ACE (Active Constellation Extension), (ix) tramas de extensión futura FEFs (Future Extension Frames), (x) eliminación de paquetes TS nulos, (xi) encapsulación GSE (Generic Stream Encapsulation) para la transmisión eficiente de contenidos IP.

El uso de los mecanismos FEC LDPC y $\mathrm{BCH}$ aumenta la robustez de la señal con respecto a DVB-T. Este aumento permite una cobertura de hasta un $70 \%$ para igual potencia que DVB-T, o un ahorro de energía para una misma cobertura [7].

En DVB-T2 la creación de múltiples canales lógicos a nivel de capa física (MPLPs), permiten la transmisión de diferentes servicios en un mismo canal RF con independiente MODCOD y entrelazado temporal para cada uno de ellos. El máximo número de PLPs en DVB-T2 es 255, aunque depende del tamaño de FFT y de la señalización L1 [8].

A diferencia de DVB-T, DVB-T2 incluye entrelazado temporal, lo que incrementa el nivel de cobertura.

La constelación 256QAM aumenta la capacidad de transmisión del sistema. Por su parte la técnica de constelaciones rotadas, que aplica un determinado ángulo de giro a todos los puntos de la constelación, aumenta la robustez de la señal transmitida. Esta técnica presenta mejores ganancias en constelaciones de orden bajo (QPSK, 16QAM) y tasas de codificación altas $(5 / 6,7 / 8)$ [6]. En DVB-T2 el uso de la técnica MISO basada en codificación Alamouti, evita desvanecimientos de la señal causados por la presencia de señales con similares retardos $y$ niveles de potencia, provenientes de varios transmisores. Esto mejorará la recepción en áreas donde la cobertura se sobrepone. El inconveniente es la complejidad requerida en el receptor para el procesamiento de dicha técnica [8].

DVB-T2 usa un conjunto de símbolos con amplitud y fase, conocidos por los receptores para ecualizar las características del canal en tiempo y frecuencia. Dichos símbolos son los patrones de portadoras piloto (PPP). El estándar define ocho (PP1 a PP7) para minimizar la sobrecarga en función del tipo de recepción al que está orientado el servicio. Así en recepción fija se usarán PPP menos densos como PP1 o PP2 para aumentar la capacidad de transmisión [8].

El uso de FFT grandes $16 \mathrm{~K}$ y $32 \mathrm{~K}$ implican periodos de símbolo OFDM más largos que en DVB-T (tamaño FFT máximo 8K). Lo que aumenta la distancia entre transmisores permitiendo redes de frecuencia única $(S F N$ - Single Frecuency Network) de mayor tamaño. Las FFT grandes reducen la sobrecarga del GI y definen un mayor número de 
portadoras activas en un símbolo OFDM (modo extendido de FFT), lo que permite ganancia en capacidad del sistema, aunque el inconveniente es la reducción de la velocidad máxima soportada.

Para ampliar la cobertura o reducir el costo de operación de una red utilizando menor energía, DVB-T2 hace que el amplificador de potencia de RF trabaje al máximo nivel de salida permitido (punto de saturación) mediante el uso del mecanismo PAPR, para lo cual utiliza dos técnicas; TR para constelaciones mayores y ACE (no compatible con constelaciones rotadas) para constelaciones de orden bajo.

Las tramas FEF son usadas para brindar servicios adicionales a T2 convencional. Tienen una duración máxima de $250 \mathrm{~ms}$ y son multiplexadas en tiempo con las tramas T2. A diferencia de la transmisión con MPLPs, FEF configura un modo de transmisión independiente a las tramas T2 base (incluyendo tamaño de FFT, GI y PPP), con lo cual se han definido los modos de transmisión DVB-T2 lite y DVB-NGH.

La eliminación de paquetes TS nulos, generados en transmisión para mantener un flujo MPEG TS para luego ser reinsertados en recepción, permite reutilizar el espacio ocupado por éstos con paquetes útiles de datos.

La utilización del protocolo de encapsulación GSE permite la transmisión eficiente de datos IP sobre las tramas banda base de T2, evitando los problemas generados por transportar contenido IP sobre tramas MPEG-TS (utilizado en DVB-T), como el corto tamaño de las tramas IP, la sobrecarga de señalización y la utilización de un protocolo adicional de encapsulación; MPE (Multi Protocol Encapsulation).

\section{DISEÑO DEL ESTÁNDAR DVB-RCT2}

Para resolver el problema de acceso a Internet en zonas rurales de América Latina, se propone el estándar DVBRCT2, siendo una propuesta innovadora que podría ser utilizada con cualquier tecnología de TDT para el DL.

\section{A. Elementos de DVB-T2}

DVB-RCT2 utilizará las siguientes funcionalidades de DVB-T2, (i) mecanismos de corrección de errores LDPC+BCH, (ii) constelación 256QAM y constelaciones rotadas, (iii) entrelazado temporal, (iv) tamaños de FFT $16 \mathrm{~K}$ y $32 \mathrm{~K}$, con los modos de portadoras extendidos, (v) patrones de portadoras piloto PP1 a PP7, (vi) protocolo de encapsulación GSE, (vii) tramas de extensión futura FEFs.

La incorporación de los mecanismos FEC de DVB-T2 permitirá mayor cobertura para una misma capacidad, o mayor capacidad para una misma cobertura. Además la posibilidad de decodificar esquemas de modulación de mayor orden con un mismo nivel de CNR, debido al rendimiento de los algoritmos LDPC $+\mathrm{BCH}$ comparado con los turbo códigos de DVB-RCT para las mismas condiciones de recepción.

El uso de la constelación 256QAM aumentará la capacidad de transmisión para los usuarios con buenas condiciones de recepción, o para que con la misma capacidad se consuma menos recursos.

El uso de constelaciones rotadas aumentará la robustez de la señal en las ráfagas que utilicen más de una portadora.
El uso de entrelazado temporal, mejoraría la robustez de la transmisión, implicando una estructura de trama especial, por ejemplo al reducir el número de bloques TI dentro de una trama banda base se incrementaría la capacidad de transmisión; y al aumentarlo se reduciría el tiempo de TI. Sin embargo para recepción fija la ganancia no es significativa.

Los tamaños FFT $16 \mathrm{~K}$ y $32 \mathrm{~K}$ mejorarán el rendimiento de la redes SFN y el uso de los modos extendidos de FFT aumentará la capacidad de transmisión. Por esto en RCT2 se utilizará para recepción fija FFT 32KE, con el uso de PP7.

Las tramas FEF se utilizarán para compartir el canal RF entre las transmisiones UL y DL (siempre y cuando se utilice DVB-T2 en el DL). Esta posibilidad no existe en DVB-RCT.

Ya que con RCT2 se brindará conectividad a Internet es importante que provea contenidos IP, reduzca la sobrecarga de encapsulamiento y permita mejor capacidad de transmisión. Por ello se utilizará la transmisión de IP sobre el protocolo GSE, para encapsular el datagrama IP sobre la capa de enlace. Además la cabecera GSE necesita de 7 a 10 bytes por PDU, en comparación con los 2 bytes para la cabecera MPE, 4 bytes del control de redundancia (CRC) por PDU y 4 bytes de la cabecera TS por cada 184 bytes de carga útil TS que se necesitaría para la opción de IP sobre MPE de DVB-T. En este sentido la sobrecarga de encapsulamiento depende del tamaño de la PDU, pero aproximadamente un $70 \%$ de reducción se conseguiría con la opción de RCT2 de forma parecida a como se propuso para DVB-NGH (Next Generation Handheld) [9].

\section{B. Elementos de DVB-RCT}

De DVB-RCT se propone adoptar para DVB-RCT2 las siguientes características, (i) tramas de transmisión (TF1, TF2), (ii) estructuras de ráfaga (BS1, BS2, BS3), (iii) conformado de señal Nyquist y rectangular, (iv) esquemas de acceso al medio (sinronización, MAS1, MAS2, MAS3).

DVB-RCT2 usará las combinaciones permitidas de las dos TF con las tres BS para definir los MAS, tal como se hace en DVB-RCT. Además en DVB-RCT2 los modos de transmisión aumentarán de 6 a 27 debido a la combinación de los tamaños de FFT de DVB-T2 con las CS de DVB-RCT, pudiendo por usuario utilizar solo un modo a la vez. A nivel de capa física RCT2 podrá utilizar en general los conformados de señal Nyquist y rectangular, pero en este artículo para proveer de conectividad a zonas rurales se utilizará el conformado Nyquist, BS3 y el MAS de sincronización.

Se utiliza conformado de Nyquist, debido a que los usuarios se encuentras dispersos unos de otros, así como de la estación base. El uso de BS3 es porque el protocolo MAC del estándar 802.16a (Wimax) que está basado en OFDMA usa una capa física similar a la de DVB-RCT usando esta estructura de ráfaga. Y BS3 porque cuenta en UL con 59 subcanales de los cuales para mejorar el rendimiento del sistema se utilizarán 5 para formar el subcanal de sincronización y los 54 restantes serán para transmisión de datos. Además se utilizará el modo de acceso de sincronización por su notable rendimiento para servicios con altas y bajas tasas de datos con respecto al modo de acceso de contención [10]. El principal beneficio del modo de sincronización es la reducción de las colisiones (presentes en el subcanal de sincronización, más no en el de datos), 
TABLA II. PARÁMETROS DE DVB-RCT Y DVB-RCT2

\begin{tabular}{|c|c|c|}
\hline & DVB-RCT & DVB-RCT2 \\
\hline $\begin{array}{l}\text { Conformados de } \\
\text { tráfico }\end{array}$ & Nyquist, Rectangular & Nyquist, Rectangular \\
\hline $\begin{array}{l}\text { Esquemas de } \\
\text { Modulación }\end{array}$ & QPSK, 16QAM, 64QAM & $\begin{array}{l}\text { QPSK, 16QAM, } \\
\text { 64QAM, 256QAM }\end{array}$ \\
\hline FEC & $\begin{array}{c}\text { Cod. Convolucional }+ \\
\text { Reed Solomon } \\
1 / 2,3 / 4 \\
\end{array}$ & $\begin{array}{c}\text { LDPC + BCH } \\
1 / 2, \mathbf{3 / 5}, \mathbf{2 / 3}, 3 / 4, \mathbf{4 / 5}, \mathbf{5} / \mathbf{6}\end{array}$ \\
\hline GI & $1 / 4,1 / 8,1 / 16,1 / 32$ & $\begin{array}{c}1 / 4, \mathbf{1 9} / \mathbf{1 2 8}, 1 / 8, \mathbf{1 9} / 256,1 / 16, \\
1 / 32, \mathbf{1} / \mathbf{1 2 8}\end{array}$ \\
\hline Tamaño de FFT & $1 \mathrm{~K}, 2 \mathrm{~K}$ & $1 \mathrm{~K}, 2 \mathrm{~K}, \mathbf{4 K}, \mathbf{8 K}, \mathbf{1 6 K}, \mathbf{3 2 K}$ \\
\hline PPP & PP1 & PP1 a PP7 \\
\hline $\begin{array}{c}\text { Entrelazado } \\
\text { temporal }\end{array}$ & $\begin{array}{c}\text { Robustez frente a } \\
\text { interferencia impulsiva }\end{array}$ & En tramas banda base \\
\hline $\begin{array}{c}\text { Constelaciones } \\
\text { rotadas }\end{array}$ & No usa & Sí \\
\hline FEF & No usa & Sí \\
\hline $\begin{array}{l}\text { Transmisión de } \\
\text { Contenidos IP }\end{array}$ & $\mathrm{IP} \rightarrow \mathrm{MPE} \rightarrow \mathrm{TS}$ & $\mathrm{IP} \rightarrow \mathrm{GSE}$ \\
\hline $\begin{array}{l}\text { Tramas de } \\
\text { transmisión }\end{array}$ & $\mathrm{TF} 1, \mathrm{TF} 2$ & TF1, TF2 \\
\hline $\begin{array}{c}\text { Estructuras de } \\
\text { ráfaga }\end{array}$ & $\mathrm{BS} 1, \mathrm{BS} 2, \mathrm{BS} 3$ & $\mathrm{BS} 1, \mathrm{BS} 2, \mathrm{BS} 3$ \\
\hline $\begin{array}{c}\text { Esquemas de } \\
\text { acceso al medio }\end{array}$ & $\begin{array}{c}\text { Sincronización, MAS1, } \\
\text { MAS2, MAS3 } \\
\end{array}$ & $\begin{array}{c}\text { Sincronización, MAS1, } \\
\text { MAS2, MAS3 }\end{array}$ \\
\hline
\end{tabular}

permitiendo además asignar ancho de banda a los RCTTs. La Tabla II resume los parámetros de diseño descritos.

\section{Evaluación Inicial de Prestaciones DVB-RCT2}

\section{A. Ganancias en Cobertura y Capacidad con respecto a} $D V B-R C T$

DVB-RCT2 proporciona ganancia en capacidad o cobertura similar a las ganancias de DVB-T2. Esto es hasta un 70\% de capacidad para la misma cobertura y $5 \mathrm{~dB}$ para una misma capacidad. DVB-RCT2 también proporciona una capacidad de transmisión mayor tanto en DL como en UL, es así que en DL se tendrá 37,88 Mbps para cada usuario en un ancho de banda de $6 \mathrm{MHz}$ (FFT 32KE, GI 1/128, 256QAM5/6, PP7); mientras que la capacidad máxima de DVB-RCT en el mismo ancho de banda es de 19,21 Mbps (FFT 2K, GI 1/32, 64QAM 7/8). Por otra parte en UL la capacidad de transmisión de un usuario en DVB-RCT2 será de 5,64 Mbps, comparado con los 4,26 Mbps provistos por DVB-RCT, considerando en ambos casos ancho de banda de $6 \mathrm{MHz}$ (utilizado en Latinoamérica para transmitir los canales de TV en TDT) y MODCOD QPSK $1 / 2$.

La mínima CNR de DVB-RCT2 para recepción fija (modelada por un canal Rice con visión directa) es de 2,7 dB, mientras que este valor para DVB-RCT es de 4,6 dB considerando en ambos casos QPSK $1 / 2$.

DVB-RCT2 permite mayores velocidades de transmisión que DVB-RCT (considerando la misma cobertura/CNR), lo que implica que los usuarios utilicen menos recursos para transmitir la misma cantidad de información. Además, con DVB-RCT2 se podrían tener celdas más grandes debido a la ganancia de cobertura.

Como se comentó, DVB-RCT está basado en DVB-T, mientras que DVB-RCT2 lo está con DVB-T2, razón por la cual las capacidades de transmisión de la tecnología propuesta son mayores que la de su predecesora DVB-RCT. En la Fig. 2, se observa una comparativa entre DVB-T y DVB-T2 en términos de capacidad de transmisión en función de la CNR.

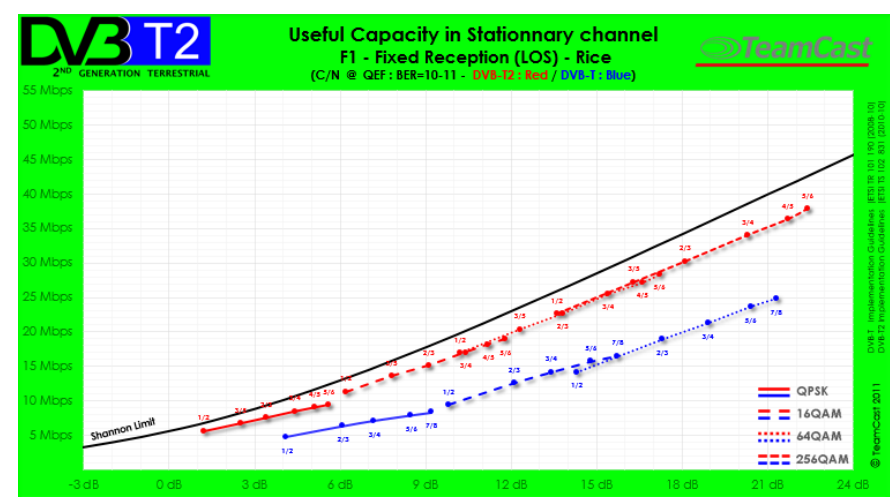

Figura 2. Rendimiento de los estándares DVB-T y DVB-T2 para un canal de 6 $\mathrm{MHz}$, transmisión DL [11].

\section{Estimación de Planificación de Redes de TDT}

\section{A. Cálculo del Balance de Enlace}

El balance de enlace establece las ganancias y pérdidas entre el transmisor y receptor en un sistema de telecomunicaciones. Para DVB-RCT2 se han utilizado las fórmulas definidas en [6] y [13], en donde la potencia recibida $\left(P_{r x}\right)$ se estima mediante la ecuación 1:

$P_{r x}=P_{t x}+G_{t x}-L_{f t x}-L_{r u r a l}+G_{r x}-L_{f r x}$

Donde $P_{t x}$ es la potencia transmitida por la estación base, $G_{t x}$ y $G_{r x}$ representan la ganancia de la antena transmisora y receptora (para la antena receptora se asume recepción fija con una antena directiva con ganancia $11 \mathrm{dBd}$ ). En este apartado se utilizó además un factor de corrección, debido a la utilización de frecuencias diferentes a las de referencia. $L_{f t x}$ y $L_{f x x}$ son las pérdidas en los cables desde los amplificadores hasta las antenas (para los cables del receptor, se asume $4 \mathrm{~dB}$ ). $L_{\text {rural }}$ son las pérdidas de propagación en escenario rural.

\section{B. Intensidad de campo mínimo $\left(E_{\min }\right)$}

En la ecuación 2 se calcula el nivel de potencia de ruido utilizado para encontrar la intensidad de campo mínimo.

$P_{n}=N F+10 \cdot \log _{10}\left[K T_{0} B\right]$

Donde $N F$ es la figura de ruido del receptor (Tabla III); $K$ es la constante de Boltzman (1.3806504x10-23Ws/K); $T_{0}$ es la temperatura absoluta (290K); $B$ es el ancho de banda $\left(5,78 \cdot 10^{6}\right.$ $\mathrm{Hz}$, ancho de banda útil en un canal de $6 \mathrm{MHz}$.

La ecuación 3 define el valor medio de intensidad de campo mínimo a la entrada del receptor para hacer la estimación de cobertura.

$E_{\text {min }}=P n+C N R-A a+L f t x+L f r x+C o r r+C l+L d-G d$ $+G C+120+10 * \log 10(120 * \pi)$

Donde $P_{n}$ es el nivel de potencia de ruido; $C N R$ el mínimo nivel requerido para un determinado modo de transmisión; $A_{a}$ representa la apertura efectiva de la antena (se utiliza la ganancia de la antena que depende de la frecuencia utilizada); Corr es el factor de corrección en función de la frecuencia; $C_{l}$ es el factor de corrección de ubicación, que depende de la probabilidad de ubicación (por ejemplo $70 \%$ o $95 \%$ ); $L_{d}$ y $G_{c}$ son las pérdidas en el duplexor y la ganancia de concentración en el terminal de usuario (esto solo afecta la UL); y $G_{d}$ es la ganancia de diversidad en la estación base. 
TABLA II. BALANCE DE ENLACE PARA ESCENARIO RURAL EN DL Y UL

\begin{tabular}{|c|c|c|c|c|}
\hline & \multicolumn{2}{|c|}{ Balance de enlace en DL } & \multicolumn{2}{|c|}{ Balance de enlace en UL } \\
\hline PARÁMETRO & ISDB-Tb & DVB-T2 & DVB-RCT & DVB-RCT2 \\
\hline $\begin{array}{l}\text { Figura de ruido } \\
\text { del receptor }\end{array}$ & $7 \mathrm{~dB}[12]$ & $6 \mathrm{~dB}[6]$ & $0 \mathrm{~dB}[5]$ & $\begin{array}{c}0 \mathrm{~dB} \\
\text { basado en } \\
\text { DVB-RCT }\end{array}$ \\
\hline Frecuencia & \multicolumn{2}{|c|}{$\begin{array}{c}513,25 \mathrm{MHz} \rightarrow \text { canal } 21 \\
\text { para DL } \\
(\text { Corr }=0,11 \mathrm{~dB})\end{array}$} & \multicolumn{2}{|c|}{$\begin{array}{c}501,25 \mathrm{MHz} \rightarrow \text { canal } 19 \\
\text { para UL } \\
(\text { Corr }=0,01 \mathrm{~dB})\end{array}$} \\
\hline $\begin{array}{c}\text { Potencia del } \\
\text { transmisor } \\
\text { torre de TV } \\
\mathbf{P}_{\mathrm{T}(\mathrm{BS})}\end{array}$ & $\begin{array}{c}1 \mathrm{~kW} \\
(60 \mathrm{dBm})\end{array}$ & $\begin{array}{c}1 \mathrm{~kW} \\
(60 \mathrm{dBm})\end{array}$ & $\begin{array}{c}10,37 \mathrm{~kW} \\
(70,16 \mathrm{dBm}) \\
\text { en el borde } \\
\text { de cobertura }\end{array}$ & $\begin{array}{c}1,19 \mathrm{~kW} \\
(60,77 \mathrm{dBm}) \\
\text { en el borde } \\
\text { de cobertura }\end{array}$ \\
\hline $\begin{array}{c}\text { Potencia del } \\
\text { transmisor del } \\
\text { usuario } \mathbf{P}_{\mathrm{T}(\mathrm{RCTT})} \\
\end{array}$ & $\begin{array}{c}- \\
\text { (No usado) }\end{array}$ & $\begin{array}{c}- \\
\text { (No usado) }\end{array}$ & $\begin{array}{l}\text { Hasta } 1 \mathrm{~W} \\
(30 \mathrm{dBm})\end{array}$ & $\begin{array}{l}\text { Hasta } 1 \mathrm{~W} \\
(30 \mathrm{dBm})\end{array}$ \\
\hline $\begin{array}{c}\text { Pérdidas en el } \\
\text { duplexor } \\
L d_{(\mathrm{RCTT})}\end{array}$ & $\begin{array}{c}- \\
\text { (No usado) }\end{array}$ & $\begin{array}{c}- \\
\text { (No usado) }\end{array}$ & $4 \mathrm{~dB}$ & $4 \mathrm{~dB}$ \\
\hline $\begin{array}{c}\text { Ganancia de } \\
\text { diversidad } \\
\qquad d_{(B S)}\end{array}$ & $\begin{array}{c}- \\
\text { (No usado) }\end{array}$ & $\begin{array}{c}- \\
\text { (No usado) }\end{array}$ & $\begin{array}{c}4 \mathrm{~dB} \\
\text { para } \\
\text { compensar } \\
L d_{(R C T T)}\end{array}$ & $\begin{array}{c}4 \mathrm{~dB} \\
\text { para } \\
\text { compensar } \\
L d_{(R C T T)}\end{array}$ \\
\hline $\begin{array}{c}\text { Ganancia de } \\
\text { concentración } \\
\mathbf{G}_{\mathrm{C}(\mathrm{RCTT})} \\
\end{array}$ & $\begin{array}{c}- \\
\text { (No usado) }\end{array}$ & $\begin{array}{c}- \\
\text { (No usado) }\end{array}$ & $17,7 \mathrm{~dB}[9]$ & $\begin{array}{c}12,49 \mathrm{~dB} \text { si } \\
n=54\end{array}$ \\
\hline CNR utilizada & $\begin{array}{c}21,06 \\
\text { 64QAM 3/4 } \\
\end{array}$ & $\begin{array}{c}16,91 \\
64 \mathrm{QAM} 3 / 4 \\
\end{array}$ & $\begin{array}{c}4,6 \\
\text { QPSK 1/2 } \\
\end{array}$ & $\begin{array}{c}2,7 \\
\text { QPSK 1/2 }\end{array}$ \\
\hline $\begin{array}{c}\text { dB utilizados } \\
\text { por tecnología } \\
\text { para el balance } \\
\text { de enlace } \\
\end{array}$ & $88,17 \mathrm{~dB}$ & $66,11 \mathrm{~dB}$ & $130,47 \mathrm{~dB}$ & $113,97 \mathrm{~dB}$ \\
\hline $\mathbf{L}_{\text {rural }}$ & 128,1 & 132,25 & 138,28 & 128,89 \\
\hline $\begin{array}{c}\mathbf{E}_{\min } \text { para } \\
\text { recepción al } \\
\mathbf{7 0 \%}\end{array}$ & 46,9 & 41,9 & 40,9 & 27,6 \\
\hline
\end{tabular}

C. Diferencias en el cálculo de Balance de Enlace para tecnologías de TDT

En la Tabla III se indican los parámetros de balance de enlace para tecnologías de TDT en donde debido a las mejoras tecnológicas de los receptores, el factor de ruido en DVB-T2 es de sólo $6 \mathrm{~dB}$, aunque ISDB-Tb podría utilizar este valor.

La frecuencia actual considerada varía por la utilización de canales diferentes para DL y UL, lo que incide en la ganancia de las antenas debido al factor de corrección utilizado. Puesto que el enlace entre DL y UL no es simétrico, hay parámetros que varían como la potencia de transmisión utilizada, que en DL fue de $1 \mathrm{~kW}$; mientras que para UL se calculó la potencia máxima que debería tener la estación base en el borde del área de cobertura, considerando que la potencia los RCTTs era de 1 W [5]. Otros parámetros usados son pérdidas en el duplexor, con $0 \mathrm{~dB}$ para las tecnologías de DL (TDT normal) y $4 \mathrm{~dB}$ para las de DL y UL, debido a la existencia del duplexor en el receptor existe una ganancia de diversidad $0 \mathrm{~dB}$ para DL y 4 dB para UL. La ganancia de concentración solo es utilizada en UL puesto que al transmitir se usa una porción del canal. Para CT2 se consideró que todos los usuarios transmitían $(n=54)$.

En el caso de estudio definido para este artículo se utilizaron las tecnologías ISDB-Tb para DL (64QAM 3/4) y DVB-RCT2 para UL (QPSK 1/2), puesto que con dichos MODCODs se balanceó el enlace entre DL y UL, determinando que las pérdidas en espacio rural $\left(L_{\text {rural }}\right)$, son las mismas para las tecnologías ISDB-Tb y DVB-RCT2. Además la CNR de DVB-RCT2 es más baja con lo cual la $\mathrm{E}_{\min }$ es menor, lo que brinda mayor cobertura que DVB-RCT.

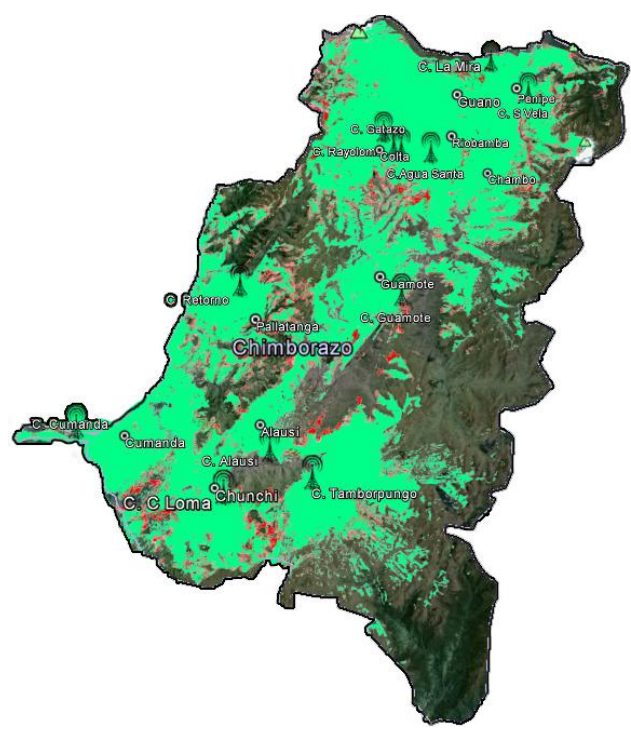

Figura 3. Cobertura DL ISDB-Tb MODCOD 64QAM 3/4, recepción al 70\% con retardos artificiales en los transmisores

\section{Escenario y análisis de cobertura e interferencia}

Los ejercicios de planificación fueron realizados mediante simulaciones con el software ICS Telecom en la provincia de Chimborazo, República del Ecuador, debido a que esta zona presenta escenarios rurales con línea de vista ( $L O S$ - Line Of Sight). Se consideró para los receptores de usuario antenas fijas en el tejado con ganancia de $11 \mathrm{~dB}$, potencia de transmisión $1 \mathrm{~W}$; y para las estaciones base sistemas radiantes directivos con arreglos de antenas diédricas de ganancia 11 $\mathrm{dB}$, potencia de transmisión $1 \mathrm{~kW}$, además de frecuencias entre los 513,25 y $561,25 \mathrm{MHz}$ para DL y $501,25 \mathrm{MHz}$ para UL. El modelo de propagación usado fue ITU-R 525, método Deygout 94 debido al área geográfica montañosa de la zona. Para la cobertura se analizó si la $E_{\min }$ y la CNR superaron el umbral mínimo requerido para un determinado MODCOD; y para las interferencias se analizó el efecto de la propagación multicamino en una red SFN. Así, un eco es constructivo o destructivo dependiendo si llega o no dentro del GI.

\section{RESULTADOS DE PLANIFICACIÓN}

En la Fig. 3 se muestran las zonas de cobertura (en verde claro) y de interferencia (en rojo) que se observarían en el DL con la tecnología ISDB-Tb para el MODCOD 64QAM 3/4 utilizando las ubicaciones de TDT en la provincia de Chimborazo. Las interferencias dependen de la combinación de GI y FFT, y pueden ser eliminadas utilizando un GI/FFT mayor, lo que además permitiría aumentar la capacidad de transmisión. Las zonas de interferencia son un porcentaje muy residual de la cobertura total y se ubican en áreas donde no tienen efecto nocivo para el servicio de radiodifusión, debido a los retardos artificiales aplicados a los transmisores de TV.

En la Fig. 4 se observan las zonas de cobertura en UL para DVB-RCT2, considerando MODCOD QPSK 1/2, asegurando de esta forma que los equipos de usuario (set-top-box) cuenten con la cobertura que les proporcionará una u otra estación base en determinada zona. En este caso, se asumió que los set-topbox que permiten la recepción de señales de TDT en DL, y a 


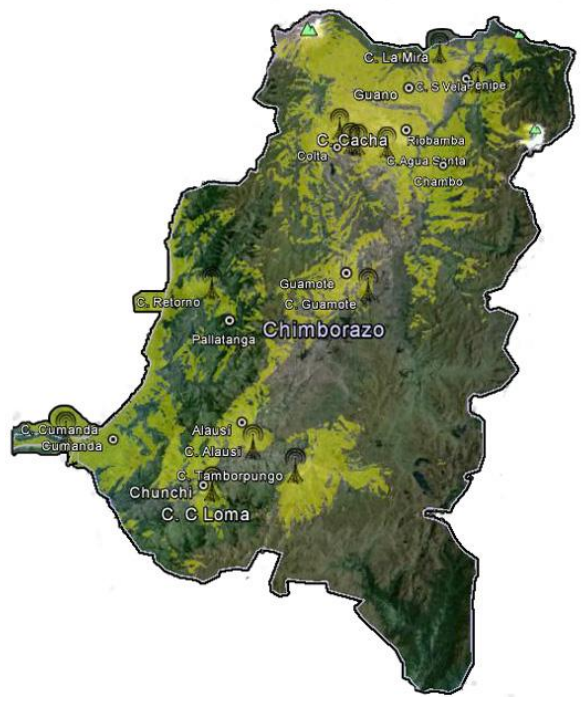

Figura 4. Cobertura UL DVB-RCT2 MODCOD QPSK $1 / 2$

su vez la transmisión de las peticiones de internet en UL, pertenecen a la misma celda y no tienen movimiento, por lo que comparten el canal de RF, no existiendo interferencias.

Por lo anteriormente mencionado, es posible hacer una red SFN ISDB-T para toda la región, dedicando un canal RF para el DL y otro para el UL, o en su defecto reutilizando frecuencias de tal modo que la misma frecuencia se usada en la región para transmitir contenidos en dos sitios diferentes (tanto en DL como en UL), pero además cuando existan pocos usuarios a ser conectados en determinada zona, se podría utilizar un solo canal de RF para DL y UL usando FEFs, con lo cual se optimizaría los canales disponibles.

\section{CONCLUSIONES}

En este artículo se ha propuesto la definición de una nueva tecnología de TDT, denominada DVB-RCT2, que permite disponer de un canal de retorno robusto y con aplicación en diversos campos como la provisión de acceso a Internet en zonas rurales. Esta alternativa ofrece un alto porcentaje de penetración y costos de despliegue más baratos con respecto a otras soluciones de conectividad, debido a que puede reutilizar infraestructura de la TV analógica y/o digital.

En este trabajo se ha definido y evaluado las prestaciones de DVB-RCT2. En particular, el canal ascendente (UL), que complementa al de radiodifusión (DL). El UL se usa para el envío de las peticiones de usuario hacia la estación base, utilizando una adaptación que mejora las prestaciones de la arquitectura RCT, mientras que en DL el sistema podría usar las arquitecturas ISDB-Tb, DVB-T o DVB-T2, permitiendo que RCT2 pueda ser usado en toda Latinoamérica.

Las prestaciones del sistema propuesto superan a las del estándar DVB-RCT tal como se ha calculado en la sección V, consiguiendo con DVB-RCT2 un porcentaje de cobertura del $82 \%$, y una capacidad máxima de transmisión de $37,88 \mathrm{Mbps}$ en DL y 5,64 Mbps en UL; comparado con el 75\%, 19,21 Mbps en DL y 4,26 Mbps en UL de DVB-RCT; lo que da una ganancia del 7\% en cobertura, 18,67 Mbps en DL, y de 1,38 Mbps en UL. La ganancia en capacidad es por la utilización del protocolo GSE, el cual reduce la sobrecarga de señalización para la transmisión de datos IP.

El despliegue de un sistema como el propuesto no supone la modificación de los actuales transmisores de TDT, ni tiene efecto nocivo sobre las emisiones en curso, pudiendo ofrecer el servicio de acceso a Internet con instalaciones de bajo coste y cobertura amplia en prácticamente todo el Ecuador.

\section{REFERENCIAS}

[1] UIT, "Informe sobre el Desarrollo Mundial de las Telecomunicaciones/TIC de 2010," Verificación de los objetivos de la CMSI, Examen Intermedio, Resumen Ejecutivo, pp. 11, Suiza 2010.

[2] A. Kholod y J. Lewis, "El dividendo digital: oportunidades y retos," Actualidades de la UIT, no. 1, pp. 1, Enero-Febrero 2010.

[3] J. Ruiz, "802.16 vs WIMAX," Tendencias, no. 156, pp. 64, Abril-Mayo 2006.

[4] DVB, "DVB-RCT Fact Sheet," Agosto 2013.

[5] ETSI, EN 301958 v1.1.1, "Digital Video Broadcasting (DVB); Interaction Channel for Digital Terrestrial Television (RCT) incorporating Multiple Access OFDM," Marzo 2002.

[6] European Broadcasting Union, "Frequency and Network Planning Aspects of DVB-T2," EBU-TECH 3348, May. 2012.

[7] L. Eizmendi, et al., "DVB-T2: The Second Generation of Terrestrial Digital Video Broadcasting System," IEEE Trans. on Broadcasting, vol. 60, no. 2, pp6-13, Junio 2014.

[8] ETSI, TS 102831 v1.2.1, "Digital Video Broadcasting (DVB); Implementation guidelines for a second generation digital terrestrial television broadcasting system (DVB-T2)," Aug. 2012.

[9] L. Konrad, D. Gómez-Barquero, C. Knuttson, y M. Petrov, "Overhead Reduction Methods in DVB-NGH," Next Generation Mobile Broadcasting, ch. 17, pp, 498-499, 2013.

[10]F. Fraile Gil y D. Gómez-Barquero, "On the Capability of DVB-RCT to provide Interactive Services," Universidad de Gävle, Suecia, Marzo 2004.

[11] TeamCast. "Universal DVB-T2 Calculator", DVB-T2 Capacity Evaluator, Diciembre 2012

[12] ABNT, “Televisión Digital Terrestre - Guía de operación Parte1: Sistema de transmisión - Guía para implementación de ABNT NBR 15601:2007”, Agosto 2008.

[13]A. C. Guedes de Carvalho Reis y P. R. de Lira Gondim, "Performance Evaluation of the DVB-RCT Standard for Interactive Services," IEEE Trans. on Broadcasting, vol. 57, no. 4, pp. 3,7,8,9, Dic. 2011.

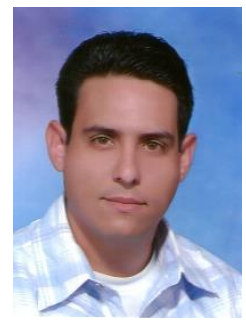

Ciro Radicelli García Se graduó de Ingeniero en Sistemas Informáticos en 2006 y de Magister en Interconectividad de Redes en 2010 en la Escuela Superior Politécnica de Chimborazo (ESPOCH), Ecuador. Obtuvo el título de Máster en Tecnologías, Sistemas y Redes de Comunicación en 2013 en la Universidad Politécnica de Valencia (UPV), España. Actualmente es estudiante de Doctorado en Telecomunicación en la UPV. Sus intereses de investigación están centrados en TDT.

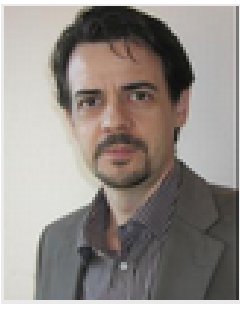

Narcis Cardona En 1990 recibió el título de máster en telecomunicaciones de la Universidad Politécnica de Cataluña y en 1995 el título de Doctor en telecomunicaciones de la UPV. Desde 1990 está vinculado con la UPV donde es profesor catedrático y director del grupo de comunicaciones móviles. El profesor Cardona ha sido vicepresidente del COST273, ha estado a cargo del WG3 del COST2100 en el área redes de acceso vía radio, y actualmente preside el EU COST IC1004.

David Gómez Barquero Doctor Ingeniero de Telecomunicación por la UPV. Es Técnico Superior de Investigación en el Instituto de Telecomunicaciones y Aplicaciones Multimedia (iTEAM) de la UPV, donde dirige un grupo de investigación sobre TDT. Desde 2008 participa en el módulo técnico del foro de estandarización DVB. Ha participado en la validación de la tecnología DVB-T2, y en el desarrollo de sus evoluciones móviles; T2-Lite y DVB-NGH. 